2015

Does verbal labeling influence age differences in proactive and reactive cognitive control?

\author{
Kray, J
}

http://hdl.handle.net/10026.1/15561

10.1037/a0038795

Developmental Psychology

American Psychological Association (APA)

All content in PEARL is protected by copyright law. Author manuscripts are made available in accordance with publisher policies. Please cite only the published version using the details provided on the item record or document. In the absence of an open licence (e.g. Creative Commons), permissions for further reuse of content should be sought from the publisher or author. 


\title{
Does Verbal Labeling Influence Age Differences in Proactive and Reactive Cognitive Control?
}

\author{
Jutta Kray, Hannah Schmitt, Sonja Heintz \\ Saarland University, Saarbrücken, Germany \\ $\&$ \\ Agnès Blaye \\ Aix-Marseille University, Marseille, France
}

Please address correspondence to:

Jutta Kray, Department of Psychology, Saarland University, Campus A 1 3, D - 66123 Saarbrücken, Germany, Phone +49 681302 3872, Fax +49 681302 6575, E-mail: j.kray@mx.uni-saarland.de

This article may not exactly replicate the final version published in the APA journal. It is not the copy of record.

http://psycnet.apa.org/journals/dev/51/3/378/

https://doi.org/10.1037/a0038795

(C) 2015 American Psychological Association, all rights reserved

\section{Reference}

Kray, J., Schmitt, H., Heintz, S., \& Blaye, A. (2015). Does verbal labeling influence age differences in proactive and reactive cognitive control? Developmental Psychology, 51, 378-391. https://doi.org/10.1037/a0038795 


\begin{abstract}
The main goal of this study was to examine whether different types of verbal labeling can influence age-related changes in the dynamic control of behavior by inducing either a proactive or reactive mode of control. Proactive control is characterized by a strong engagement in maintaining task-relevant information to be optimally prepared while reactive control is characterized by a reactivation of task-related information during responding. To investigate dynamic shifts between these control modes, we applied the AX-Continuous-Performance-Task in 2 experiments that differed in the complexity of stimuli and types of labeling in children (range $=7-10$ years), younger (range $=19-33$ years), and older adults (range $=69-83$ years $)$. We expected that labeling the cue information would promote a shift from a reactive to a proactive control mode primarily in children and older adults, while labeling the probe information would result in a shift from a proactive to a reactive control mode primarily in younger adults. Results of both experiments indicated that children, younger and older adults were equally engaged in cue processing and performed the task in a proactive manner. While cue labeling did not further promote performing the task proactively, probe labeling induced a shift to a reactive control mode, especially in children. In the first experiment, including younger children than in the second experiment, children had more problems than adults to reactivate cue information to overcome a strong response tendency. These findings support the view that verbal labeling can influence the regulation of behavior by selectively attracting attention to relevant information in a given task.
\end{abstract}

Keywords: proactive control, reactive control, verbal labeling, age differences 


\section{Does Verbal Labeling Influence Age Differences in Proactive and Reactive Cognitive}

\section{Control?}

The ability to control one's own processing and to perform goal-directed behavior is seen as a hallmark of intelligent behavior and is known to change across the lifespan. Cognitive control has generally been defined as the ability to guide thoughts and actions in accord with external and internal task goals and is particularly required in situations that involve, for instance, the maintenance of task-relevant information, the inhibition of task-irrelevant information, the flexible switching between tasks, as well as the monitoring of error and conflict information (e.g., Hofmann, Schmeichel, \& Baddeley, 2012; Miller \& Cohen, 2001; Smith \& Jonides, 1999). It is now well documented that cognitive control abilities develop relatively late in childhood (for reviews, see e.g., Garon, Bryson, \& Smith, 2008; Hughes, 2011) and decline relatively early in adulthood (e.g., West \& Schwarb, 2006) and that these changes are related to lifespan changes in prefrontal lobe functioning (e.g., Braver \& Barch, 2002; Bunge \& Zelazo, 2006). As the development of cognitive control has been shown to be predictive of later success in school and academic skills, such as the performance in complex mathematical skills (e.g., Lee et al., 2012), and as it is thought to be highly related to individual differences in fluid intelligence (Duncan, 1995), the search for useful tools and interventions improving cognitive control in children and older adults is of high importance in developmental research (see also Kray \& Ferdinand, 2013).

Another key skill of human behavior is the development of language. Language, as a unique human ability, can serve as an important self-regulatory function. It supports the planning and organization of behavior, as it has already been claimed by famous developmental psychologists, such as Vygotsky (1962) and Luria (1961). Recently, developmental researchers have begun to systematically investigate the role of inner speech and verbal labeling as tools to improve the development of different aspects of cognitive control functioning (for reviews, see 
Cragg \& Nation, 2010; Kray \& Ferdinand, 2013). For instance, it has been shown that inner speech and verbal labeling support flexible switching between tasks by enhancing: (a) the representation of tasks and its rules as claimed by the cognitive complexity and control theory suggested by Zelazo and colleagues (e.g., Zelazo \& Frye, 1997); (b) the keeping track of task order when participants have to switch between task rules without explicit task cues (i.e., they need to remember the sequence of task by themselves; e.g., Karbach, Mang, \& Kray, 2010; Kray, Eber, \& Karbach, 2008; Kray, Gaspard, Karbach, \& Blaye, 2013; Kray, Lucenet, \& Blaye, 2010); and (c) the retrieval of tasks especially when task cues are abstract or less transparent and need to be transformed in a verbal format (Chevalier \& Blaye, 2009). Importantly, a number of studies found that children and older adults showed larger benefits of verbal labeling (e.g., Kray et al., 2008; Lucenet, Blaye, Chevalier, \& Kray, 2014), suggesting that they usually use verbal labeling less spontaneously as a tool for enhancing cognitive control functioning but are able to make use of it. Most of these studies have focused on age differences in the beneficial function of verbal labeling on the maintenance and retrieval of task goals when individuals have to switch back and forth between tasks. The specific aim of the present study is to extent this research in two ways: First, we used an experimental design that allows examining age differences in the dynamic control of behavior within the same task by determining individual differences in performing a task in a proactive or reactive manner. Second, we investigated whether verbal labeling has a specific selective function by attracting the attention to a specific aspect within this task at the expense of others. Importantly, we aimed to demonstrate that different types of verbal labeling instructions can influence the dynamic control of behavior differently in younger adults as compared to children and older adults, depending on which aspect of the task the attention is attracted to by verbal labeling.

\section{Investigating the Dynamic Control of Behavior}


To investigate individual differences in proactive and reactive control, researchers have used a specific variant of the AX-continuous performance test (AX-CPT; e.g., Paxton, Barch, Storandt, \& Braver, 2006). In this task, participants saw pairs of letters presented one by one on the computer screen. The first letter was the cue and the second the probe. Participants were instructed to respond only to the probe according to the following rules: If the letter " $\mathrm{A}$ " (i.e., cue A) is followed by the letter "X" (i.e., probe X), then press the target response key (e.g., the left response key). For all other combinations of cues and probes, they were instructed to press the nontarget response key (e.g., the right response key). To bias participants toward processing the cue information and thus facilitating the updating and maintenance of this information, the frequency of cue-probe combinations varied. In $70 \%$ of the trials, subjects responded to AX trials and in the remaining $30 \%$ of trials to the other cue-probe combinations. In $10 \%$ of the trials, the cue A was not followed by the probe $\mathrm{X}$ but by another probe (i.e., probe $\mathrm{Y}$ ), termed AY trials (note that Y could be one of three different letters). In 10\% of the trials, another cue (i.e., cue B) was followed by the probe letter X, termed BX trials (note that B could be one of three different letters), and in $10 \%$ the cue B was followed by Y probes, termed BY trials.

Critical for examining the two modes of control in this task is the pattern of performance on AY and BX trials, in which interference occurs induced by the response bias of the different cue-probe combinations. Participants who are strongly engaged on processing and maintaining the cue information to be optimally prepared when the probe appears should show poor performance on AY trials, because the frequency of AX trials leads to the expectancy of the occurrence of the probe letter $\mathrm{X}$, thus biasing a target response after every presentation of the cue A. At the same time, they should show good performance on BX trials as they are prepared for the nontarget response. Hence, better performance on AY trials than on BX trials has been attributed to a proactive control mode. Conversely, if participants do not strongly engage in 
processing and maintaining the cue information, subjects have to reactivate the cue information during the appearance of the probe. As a consequence, the expectancy of AX trials will diminish, leading to a better performance on AY trials, because less interference is induced during probe presentation. At the same time, participants should show relatively poor performance on BX trials, as they have to reactivate the cue information and to overcome the response tendency toward the target response key after presentation of probe X. This type of control mode has therefore been termed reactive (for a recent review, see Braver, 2012; Braver \& Barch, 2002). Of most interest in the present study is that recent research has found age differences in adopting a proactive or reactive control mode as well as critical factors that can promote a shift between these control modes independently of age, which will be briefly summarized.

\section{Age Differences in Proactive and Reactive Control}

Most of the developmental studies so far have investigated individual differences in performance as well as in neural activity in the AX-CPT between younger and older adults. A number of studies found that younger adults indeed produced more errors on AY trials than on BX trials (e.g., Paxton et al., 2006; Paxton, Barch, Racine, \& Braver, 2008; Rush, Barch, \& Braver, 2006; but see Haarmann, Ashling, Davelaar, \& Usher, 2005) and they were also slower on AY trials than on BX trials (e.g., Haarmann et al., 2005; Paxton et al., 2006, 2008; Rush et al., 2006). These findings are in line with the view that younger adults were biased toward processing the cue and hence performed the task in a proactive manner. However, the findings are more inconsistent for older adults: For the accuracy data, studies reported (a) an increase in error rates on BX trials as compared to AY trials (Paxton et al., 2008 in Study 1) and larger errors on BX trials for older than for younger adults (Haarmann et al., 2005), (b) no significant differences in error rates on AY and BX trials (Paxton et al., 2006; Rush et al., 2006), and (c) a larger difference between AY and BX trials for younger than for older adults (Paxton et al., 2008 in Study 2). For 
the latency data, studies often found longer latencies on AY than BX trials for older adults, similar to the results obtained in younger adults, numerically (Braver, Paxton, Locke, \& Barch, 2009; Paxton et al., 2006; Paxton et al., 2008 only for long delays) and statistically (Rush et al., 2006), as well as the opposite, that is, longer latencies on BX than AY trials but only for long cueprobe intervals (Haarmann et al., 2005). Most of these studies found evidence for age differences in the performance on AY and/or BX trials, but these were often not consistently obtained in the accuracy as well as in the latency data. However, more consistent evidence that older adults have problems to overcome the response tendency and to resist interference induced by the probe letter $\mathrm{X}$ comes from recent studies of the Braver group using functional MRI (fMRI) methods (Braver et al., 2009; Paxton et al., 2008). Results of these imaging studies showed that the lateral prefrontal cortex (PFC) is activated during updating and maintaining context information in younger adults, and that older adults, in contrast, showed a reduced cue-related activity and an increased probe-related activity in this region (Braver et al., 2009; Paxton et al., 2008), in line with the view of a shift from a proactive to a reactive control mode in older age.

A few studies have also examined age differences in the AX-CPT during childhood development (Chatham, Frank, \& Munakata, 2009; Lorsbach \& Reimer, 2008, 2010). Results of these studies showed a better performance to maintain and update context information in young adults than in 12-year-olds (Lorsbach \& Reimer, 2008) and in 12-year-olds than in 9-year-olds (Lorsbach \& Reimer, 2010), suggesting a development change toward performing the task more proactively. However, it should be noted here that age differences were mainly found for conditions in which demands on maintaining and processing cue information were high, that is, in conditions in which the cue information had to be maintained over a longer period of time or when children had to apply more complex instructions in the AX-CPT (Lorsbach \& Reimer, 2010). 
Using a simple version with pictorial stimuli, Chatham et al. (2009) investigated 3.5- and 8-year-old children's performance on the AX-CPT in combination with high-resolution pupillometry. Interestingly, they found age-related differences in the cue-and probe-related pupil sizes, signaling enhanced mental effort. After cue presentation, 8-year-olds showed larger pupils than 3.5- year-olds, reflecting increased mental effort for representing and maintaining context information, while 3.5 year-olds showed larger pupils than 8-year-olds after probe presentation, indicating mental effort for reactivating the context. This finding suggests a shift in the dynamic control of behavior from a reactive to a proactive control mode during childhood development. This qualitative shift has been suggested to be one important key transition in the development of cognitive control (cf. Munakata, Snyder, \& Chatham, 2012). However, it should be noted that the authors used a simplified version of the AX-CPT with only four stimuli, which might change the characteristic of the task and the response bias, and that the pattern of results was less clear for the behavioral data. For latencies 8-year-olds showed significantly slower responses on AY trials than on BX trials, as expected from a proactive control mode, but for the accuracies no such clear pattern and age differences in proactive versus reactive control was obtained.

Taken together, there is some first evidence for a shift from a reactive to a proactive control mode during childhood development, whereas the occurrence of age differences at the behavioral level seems to be dependent on the requirements of the task. On the other end of the lifespan, empirical findings suggest a shift from a proactive to a more reactive control mode in older adults, whereas the results are more consistent at the neural than at the behavioral level.

\section{Promoting Proactive and Reactive Control}

From a developmental perspective it is not only of interest to examine age differences in these control modes, but also to determine the extent to which individuals can flexibly shift between them according to the requirements of the tasks. There is indeed some first evidence that 
shifts in control modes can be induced by different situational demands. For instance, Dreisbach (2006) found that positive affect can modulate the maintenance of cue information in the AXCPT as compared to neutral and negative affect. Other studies have investigated whether such shifts can be induced by different kinds of task instructions. For instance, Braver and colleagues (2009) examined the flexibility in adapting to both types of control modes in younger and older adults by using fMRI. In younger adults, a reactive control mode was induced by monetary penalties for poor performance that shifted attention toward probe processing. They indeed found that, relative to a nonincentive condition, cue-related activity decreased and probe-related activity increased within the same regions of the lateral PFC. Of most interest for the present study is that they induced a proactive control mode in the elderly by instructing them to use a verbal labeling strategy that shifted the attention to cue processing. For instance, subjects had to name the cue (i.e., "A" or "not A") as well as the corresponding response label that was color-coded (i.e., if "X" then press the red key). Relative to pretest performance without verbal labeling, they found an increase of cue-related activity and a decrease of probe-related activity in the brain regions of interest, suggesting a shift to a proactive control mode in the older age group (cf. Braver et al., 2009). Of note, at the behavioral level, the verbal labeling instruction led to an increase of AY errors and a decrease of BX errors. However, not only the verbal labeling instruction, but also reinstructions of the task rules as well as extended practice produced the same pattern of findings, suggesting that a number of treatments can induce a proactive control mode in the elderly (Paxton et al., 2006).

From a different perspective, Lucenet et al. (2014) have recently shown that older adults and children benefit to a certain extent from verbal labeling in a task-switching paradigm forcing the use of reactive control. However, this study did not aim at investigating age differences in the 
dynamics of control. Hence, the specific function of verbal labeling in modulating the mode of control is not clear on the basis of these findings.

\section{The Present Study}

A number of recent developmental studies indicated that verbal labeling supports different aspects of cognitive control functioning, such as the maintenance of task goals in the absence of task cues or the task retrieval when abstract cues have to be transformed in a verbal format, in situations in which individuals had to flexibly switch different tasks and their corresponding S-R rules (with task-switching paradigms or the dimensional card sorting test; cf. Cragg \& Nation, 2010; Kray \& Ferdinand, 2013). Moreover, there is also evidence that individuals with impairments in these cognitive control functions, like children and older adults, show larger verbal labeling benefits, suggesting that the use of language can serve as a compensatory tool. The specific goal of the present study is to examine whether verbal labeling also supports cognitive control functions when no switching between task goals is required and whether children and older adults still show larger verbal benefits in less demanding cognitive control tasks. Therefore, we looked for an experimental design that allows to examine the flexible control of behavior within a given task, in particular, shifts between two different kind of control modes (proactive and reaction control) and that can also be applied successfully in various age ranges across the lifespan in order to investigate age differences in shifts between these two control modes. As a starting point for our research in this area and to replicate previous findings, we decided to use a very similar AX-CPT task (letter version) and selected similar age ranges as in previous developmental studies (cf. Lorsbach \& Reimer, 2010; Paxton et al., 2006) in Experiment 1. In general, empirical evidence from behavioral and neuronal studies have indicated a shift from a reactive to a proactive control mode from childhood to young adulthood and a shift from a proactive to reactive control mode from young adulthood to old age (e.g., Braver et al., 2009; 
Chatham et al., 2009; Paxton et al., 2006, 2008). Therefore, we investigated three age groups (children, younger adults, and older adults), and we expected that younger adults should show longer latencies and higher error rates on AY trials as compared to BX trials and the noninterference trials ( $\mathrm{AX}$ and $\mathrm{BY}$ ). Because behavioral findings are somewhat mixed regarding age differences, these effects may be reduced for children and older adults, while longer latencies and higher error rates on $\mathrm{BX}$ trials than on $\mathrm{AY}$ trials would suggest a reactive control mode in children and older adults.

The review of literature also indicated that the occurrence of age differences in proactive versus reactive control seems to vary with task complexity (e.g., Lorsbach \& Reimer, 2010). Therefore, and to enhance the generalization of our findings to other context situations, in particular to other kinds of stimuli and verbal labeling conditions, we ran a second experiment in parallel to the first one. The second experiment included comparable age groups with a similar AX-CPT but using quite different stimuli (male and female faces) and naming conditions (for details, see Method of Experiment 2).

The main idea of the present study was to examine whether verbal labeling has a specific selective function within a given task by attracting the attention toward processing of some information during task performance at the expense of some other information. To this end, we compared the performance in the AX-CPT between three conditions. The first condition was a control condition, in which no verbalization was required. In the second condition, termed cue labeling condition, participants were to name the cue (i.e., verbalize the cue A as "A," and the nontarget cues B as "No"). In the third condition, termed probe labeling condition, they were to name the probe by verbalizing the target probe " $\mathrm{X}$ " and otherwise saying aloud "No." Hence, we expected that cue labeling attracts the attention toward the processing of the cue information and by this supporting a proactive control mode. Therefore, we predicted a larger increase of errors 
and latencies on AY trials than on BX trials under cue labeling as compared to the control condition. Conversely, we expected that probe labeling attracts the attention toward the processing of the probe so that subjects are less engaged in cue maintenance and need to reactivate the cue information on $\mathrm{BX}$ trials to overcome the strong response tendency of the target response. Here we predicted a larger increase of errors and latencies on BX trials than on AY trials under probe as compared to the control condition. As the additional verbal labeling instruction during task performance can be seen as a secondary task, the increase of errors and latencies can also be due to a general increase in dual-task demands. Therefore, we also analyzed whether the increase under cue and probe labeling is larger as compared to the respective other labeling condition.

Regarding age differences, we expected that if children and older adults perform the task in a reactive manner as mainly shown in previous studies (e.g., Braver et al., 2009; Lorsbach \& Reimer, 2010; Paxton, 2011; Paxton et al., 2006), then cue labeling should promote a shift toward a proactive control mode. Conversely, probe labeling should induce a shift from a proactive to a reactive control mode, primarily in younger adults. Finally, we expected to observe similar results in both experiments, as control processes should be relatively independent of task-specific processes, such as type of stimuli or task-relevant verbalization.

\section{Experiment 1}

\section{Method}

Participants. Overall, 20 children, 20 younger adults, and 21 older adults participated in the first experiment. We had to exclude nine participants (two children, two younger adults, and five older adults) because their error rates were larger than three standard deviations above their corresponding group mean, resulting in a final sample of 18 children (mean age $=7.2$ years, age range $=7-8$ years), 18 younger adults (mean age $=22.5$ years, age range $=19-30$ years), and 16 
older adults (mean age $=74.4$ years, age range $=69-83$ years). Children and older adults were drawn from a participant pool of the Saarland University, and students were recruited in seminars and lectures at the Saarland University. All subjects were paid $8 €$ or received course credit as compensation for their participation. We obtained informed consent and demographic information from all subjects. All of the participants were German native speaker and reported themselves in good mental health (children: $M=1.2, S D=.38$; younger adults: $M=1.8, S D=$ .92 ; older adults: $M=2.1, S D=.57$ ) as well as physical health (children: $M=1.8, S D=.94$; younger adults: $M=1.9, S D=1.13$; older adults: $M=2.3, S D=.60)$. Mental and physical health was a self-report measure that was rated on a 5-point scale (ranging from $1=$ very good to $5=$ poor). Moreover, younger and older adults did not differ in formal years of education $(p=.19)$. Characteristics of the final sample, including performance on intellectual control variables, are displayed in Table 1.

\section{Insert Table 1 about here}

As control variables, we administered three intellectual psychometric tests: The Digit Symbol Substitution Test (cf. Lindenberger, Mayr, \& Kliegl, 1993) measured speed of processing, the Backward Digit-Span Task (cf. Wechsler, 2003) measured working-memory span, and the Spot-a-Word test (cf. Lindenberger, Mayr, \& Kliegl, 1993) measured vocabulary ${ }^{1}$. As expected from the two-component model of intellectual abilities across the lifespan (e.g., Baltes, Staudinger, \& Lindenberger, 1999), assuming differential age trends for fluid (here speed of processing and working memory) and crystallized (here vocabulary) intellectual abilities, we found age differences in all control variables, for speed of processing, $F(2,49)=100.8, p<.001$, $\eta_{\mathrm{p}}^{2}=.804$, for working memory span, $F(2,49)=39.4, p<.001, \eta_{\mathrm{p}}^{2}=.617$, and for vocabulary, 
$F(2,49)=350.8, p<.001, \eta_{\mathrm{p}}{ }^{2}=.935$ (see Table 1$)$. Planned age contrasts showed that older adults had a lower score in the speed of processing test than younger adults, $F(1,49)=51.3, p<$ $.001, \eta_{\mathrm{p}}^{2}=.511$, but significantly higher scores than children, $F(1,49)=43.7, p<.001, \eta_{\mathrm{p}}^{2}=$ .471. The same pattern was found for the working-memory span. Older adults had lower working-memory span scores than younger adults, $F(1,49)=17.7, p<.001, \eta_{\mathrm{p}}^{2}=.265$, but significantly higher scores than children, $F(1,49)=19.5, p<.001, \eta_{\mathrm{p}}^{2}=.284$. As expected, older adults performed better in the vocabulary test than younger adults, $F(1,49)=43.7, p<.001, \eta_{\mathrm{p}}^{2}=$ .471 , and children, $F(1,49)=638.1, p<.001, \eta_{\mathrm{p}}^{2}=.929$. Hence, a U-shaped pattern of age differences across the lifespan was obtained for the fluid measures, while a linear increase was obtained for the crystallized measure of intelligence (cf. Baltes et al., 1999).

Materials and procedure. Participants performed the standard AX-CPT, in which letters were presented on a 19-in. LCD screen, centered in black font (Calibri bold 48) on a white background. The experimental task was programmed in E-Prime 2.0.

In the AX-CPT (cf. Paxton et al., 2006) participants saw pairs of letters successively presented on the computer screen. The first letter was the cue and the second letter was the probe. Responses were given after the presentation of probes. If the probe was the letter " $\mathrm{X}$ " (target probe) that followed the cue letter "A," then subjects were to press the right response key (the pkey on the keyboard). If the probe was a nontarget letter (M, U, or C; generally termed "Y") that followed the target cue or a nontarget cue $(\mathrm{G}, \mathrm{S}$, or F), then they were to press the left response key (the q-key on the keyboard). Thus, there were four trial-type combinations, with AX combinations occurring in $70 \%$ of the time and each of the other three combinations (AY, BX, BY) in $10 \%$ of the time. Participants were instructed to perform the task as fast and as accurate as possible. If subjects did not respond in time, feedback appeared on the screen to respond faster on the next trial. 
To examine the influence of cue versus probe labeling on age differences in proactive and reactive control, we compared the subjects' performance across three types of experimental conditions. The first condition served as control condition in which no verbalization was required. As described earlier, the other two conditions required the participants to verbalize either the cue or the probe.

Procedure. Participants were tested individually in one session that took approximately 60 minutes. At the beginning of each session, the participants received a short demographic questionnaire and filled in an informed consent. We then administered the three psychometric tests that served as control variables (see Participants section).

Finally, participants performed the AX-CPT that lasted about 30 min. Participants worked through six blocks of 50 trials each, yielding a total of 300 trials. There were two consecutive blocks for each of the three verbalization conditions (control, cue labeling, probe labeling), while the sequence of the three conditions were counterbalanced across subjects using a Latin square design. Before each labeling condition, participants also received a demonstration of the trial procedure by the experimenter and then they performed a block of 20 practice trials without trialbased feedback to familiarize them with the AX-CPT and the labeling task.

The trial procedure in the AX-CPT was as follows: Each letter (cue and probes) was presented for $500 \mathrm{~ms}$. The time interval between cue and probe and between probe and response was fixed to $1,500 \mathrm{~ms}$ and $1,300 \mathrm{~ms}$, respectively. The response deadline was set to $1,300 \mathrm{~ms}$.

Data analysis. Practice trials were excluded for data analysis. The analysis of latencies was based on correct reaction times (RTs), and RTs faster than $180 \mathrm{~ms}$ were excluded $(0.4 \%$ of trials). Error analysis included omissions and incorrect responses².

Data were analyzed with two separate mixed ANOVA designs using the statistical package SPSS version 19. To examine age differences in proactive and reactive control and to 
compare the results of age differences in the AX-CPT with other developmental studies, we first run an ANOVA including only trials of the control condition with the between-subjects factor Age Group (children, younger adults, older adults) and the within-subjects factor Trial Type (AX, AY, BX, BY). As we were specifically interested in whether children and older adults perform the task in a more reactive control mode than younger adults, we specified two age contrasts: The first one tested for differences in mean performance between children and younger adults, and the second contrast tested for differences in mean performance between younger and older adults. Moreover, we also prespecified two a-priori contrasts for the factor Trial Type that were of main theoretical interest according to our predictions. The first contrast tested interference effects by comparing mean performance on interference trials $(\mathrm{AY}, \mathrm{BX})$ with noninterference trials $(\mathrm{AX}$, $\mathrm{BY})$, and the second one compared the performance on AY trials with the performance on BX trials.

In the second ANOVA we focused on the effects of cue and probe labeling on age differences in proactive and reactive control and therefore included only AY and BX trials. The ANOVA design included the between-subjects factor Age Group (children, younger adults, older adults) and the within-subjects factors Trial Type (AY, BX) and Verbalization (control, cue labeling, probe labeling). Age effects were again tested with the two prespecified contrasts as used in the first ANOVA design. The effects of verbal labeling were also determined by a-priori contrasts. The first one compared the mean performance in the control condition with the cue labeling condition, the second contrast mean performance in the control condition with the probe labeling condition, and the third one compared the cue labeling with the probe labeling condition in order to control for dual-task demands related to the additional labeling requirement.

\section{Results}

Age differences in proactive and reactive control. The means and standard errors of the 
mean (SEMs) of error rates and RTs are displayed in Figure 1 as a function of the three age groups and the four trial types.

\section{- Insert Figure 1 about here -}

Errors. Results on the basis of error rates revealed that children made more errors than younger adults, $F(1,49)=53.1, p<.001, \eta_{\mathrm{p}}^{2}=.520$, while younger and older adults did not differ in their error rates, $F(1,49)=2.9, p=.093, \eta_{\mathrm{p}}{ }^{2}=.056$. Participants committed more errors on interference trials (AY and BX trials) than on non-interference trials (AX and BY trials), $F(1$, $51)=29.7, p<.001, \eta_{\mathrm{p}}^{2}=.368$, and this effect was more pronounced for children than for younger adults, $F(1,49)=27.5, p<.001, \eta_{\mathrm{p}}{ }^{2}=.359$. As can be seen in Figure 1a, all participants made more errors on AY trials than on BX trials, $F(1,51)=9.5, p<.01, \eta_{\mathrm{p}}^{2}=.157$, and there was no significant difference in this effect between children and younger adults, $F(1,49)=3.3, p$ $=.077, \eta_{\mathrm{p}}^{2}=.063$, or between younger and older adults, $F(1,49)=0.0, p=.913, \eta_{\mathrm{p}}^{2}=.000$. Thus, younger adults, but also children and older adults, showed higher error rates on AY than BX trials, suggesting that they performed the task in a proactive control mode.

RTs. In line with the results on error rates, children responded slower than younger adults, $F(1,49)=131.9, p<.001, \eta_{\mathrm{p}}^{2}=.729$, and older adults responded slower that younger adults, $F(1$, $49)=12.9, p<.01, \eta_{\mathrm{p}}{ }^{2}=.208$. Latencies were slower on interference trials (AY and BX trials) than on noninterference trials ( $\mathrm{AX}$ and $\mathrm{BY}$ trials), $F(1,51)=113.4, p<.001, \eta_{\mathrm{p}}{ }^{2}=.690$, and this effect was more pronounced for children than for younger adults, $F(1,49)=8.8, p<.01, \eta_{\mathrm{p}}{ }^{2}=$ .152. Participants also responded slower on AY than BX trials, $F(1,51)=176.1, p<.001, \eta_{\mathrm{p}}{ }^{2}=$ .775 , and this effect was larger for children than for younger adults, $F(1,49)=5.5, p<.05, \eta_{\mathrm{p}}{ }^{2}=$ .101. Again, the expected slowing of responses on BX trials for children and older adults, indicating a reactive control mode, was not observed (see Figure 1b).

We also analyzed the RT data on the basis of log-transformed RTs to look at age 
differences in proportional performance differences across conditions. All results remained unchanged except one: The larger interference effect for children as compared to younger adults was no longer reliable, $F(1,49)=0.1, p=.828, \eta_{\mathrm{p}}^{2}=.001$.

The effects of cue and probe labeling. The means and SEMs for error rates and RTs are shown in Figure 2 as a function of labeling condition (control, cue labeling, probe labeling) and trial type (AY, BX) separately for each age group (children, younger adults, older adults).

\section{- Insert Figure 2 about here -}

Errors. As can be seen in Figure 2a, error rates were increased under cue labeling as compared to the control condition, especially in older adults. This observation was confirmed by the corresponding ANOVA contrasts. Error rates were significantly higher under cue labeling than under the control condition, $F(1,51)=8.5, p<.01, \eta_{\mathrm{p}}{ }^{2}=.143$, and this increase was more pronounced for older adults than for younger adults, $F(1,49)=9.7, p<.01, \eta_{\mathrm{p}}^{2}=.165$. However, this increase in error rates was not larger on AY trials than on $\mathrm{BX}$ trials, $F(1,51)=0.3, p=.621$, $\eta_{\mathrm{p}}{ }^{2}=.005$, suggesting that the cue labeling did not specifically induce AY errors, and hence, a more proactive control mode.

As can also be seen in Figure 2a, error rates were also higher under probe labeling as compared to the control condition, $F(1,51)=17.9, p<.01, \eta_{\mathrm{p}}{ }^{2}=.259$, and again this effect was more pronounced for older than younger adults, $F(1,49)=7.9, p<.01, \eta_{\mathrm{p}}^{2}=.139$. The increase of errors on $\mathrm{BX}$ trials was significantly larger than on $\mathrm{AY}$ trials, $F(1,51)=7.4, p<.01, \eta_{\mathrm{p}}{ }^{2}=$ .127 , but we obtained no interactions with the two respective age contrasts, $F(1,49)=1.5, p=$ $.234, \eta_{\mathrm{p}}^{2}=.029$, and $F(1,49)=2.5, p=.117, \eta_{\mathrm{p}}^{2}=.049$, suggesting no age differences in the increase of BX errors under probe labeling conditions.

Most critical for the interpretation of findings is the interaction between the type of labeling (cue vs. probe labeling) and trial type (AY vs. BX). The respective contrast was indeed 
significant, $F(1,51)=7.2, p<.05, \eta_{\mathrm{p}}^{2}=.124$. Separate analyses for AY and $\mathrm{BX}$ trials showed an increase of errors on $\mathrm{BX}$ trials under probe labeling compared with cue labeling, $F(1,51)=8.7, p$ $<.01, \eta_{\mathrm{p}}{ }^{2}=.145$, but no effect on AY trials, $F(1,51)=0.6, p=.450, \eta_{\mathrm{p}}{ }^{2}=.011$. Moreover, the probe labeling effect on $\mathrm{BX}$ trials was more pronounced for children than for younger adults, $F(1$, $49)=4.7, p<.05, \eta_{\mathrm{p}}{ }^{2}=.087$. Thus, probe labeling lead to more errors on BX trials, especially in children (see Figure 2a).

$\boldsymbol{R T S}$. Results revealed longer latencies under cue labeling and probe labeling than under the control condition, $F(1,51)=19.0, p<.001, \eta_{\mathrm{p}}^{2}=.271$, and $F(1,51)=69.9, p<.001, \eta_{\mathrm{p}}^{2}=$ .578 , respectively. In contrast to the error analyses, latencies did not differ significantly for cue and probe labeling conditions, $F(1,51)=0.1, p=.809, \eta_{\mathrm{p}}{ }^{2}=.001$, suggesting that the results on error rates were not simply due to a speed-accuracy trade-off. No further interactions with trial type or age were significant.

\section{Discussion of Experiment 1}

Results of the first experiment showed that younger adults produced more errors and responded slower on interference trials (AY and $\mathrm{BX}$ ) than on noninterference trials (AX and $\mathrm{BY}$ ) and also made more errors and were particularly slowed on AY trials than on BX trials. These findings suggest that younger adults were strongly engaged in cue processing and were biased toward the target response. Hence, strong interference occurred on AY trials when they had to respond to nontargets, resulting in higher error rates and slower responding. This finding is in line with most of the previous results reported in the literature and suggests that young adults perform the task in a proactive manner (Paxton et al., 2006, 2008; Rush et al., 2006; but see Haarmann et al., 2005).

Although our findings indicated that children generally made more errors on interference trials than noninterference trials, the expected increase in error rates or slower responding on $\mathrm{BX}$ 
relative to AY trials, suggesting a reactive control mode, was not obtained in the first experiment. So far, only few studies examined age differences in shifts from a reactive to a proactive control mode during late childhood (Lorsbach \& Reimer, 2010). In that study, 9-year-olds also showed larger errors rates on AY trials than on AX trials, but an increase in latencies on BX trials. However, the cue-probe delay was substantially longer in that study as compared to the present one. Finally, older adults, like children, did not significantly differ from the pattern of findings on AY and BX trials as compared to younger adults. Instead, recent aging studies reported mixed results and often failed to show age differences in this pattern across both latency and error data (e.g., Paxton et al., 2008; Rush et al., 2006), suggesting that the occurrence of age differences in shifts between control modes at the behavioral level is dependent on specific demands in the AXCPT task. This aspect will be discussed in more detail in the general discussion section.

Regarding the effects of verbal labeling, the present study aimed to gain new insights on the function of language on cognitive control functioning. In the present task, we expected that cue versus probe labeling attracts the attention to either performing the task in a more proactive or reactive manner. Results of the first experiment showed that cue labeling, although leading to higher error rates and longer latencies on AY trials as compared to the control condition, did not differentially influence task performance on AY and BX trials. Conversely, probe labeling resulted in a larger increase of errors and latencies on $\mathrm{BX}$ trials than on $\mathrm{AY}$ trials compared to the control and the cue labeling condition, especially in children. Hence, probe labeling lead to a stronger interference on trials in which the "cue B" information had to be reactivated in order to overcome the bias of the frequent target response to $\mathrm{X}$, in line with a shift toward a more reactive control mode that was more pronounced for children than for younger adults.

\section{Experiment 2}


We ran a second experiment in parallel to the first in order to test the generalization of the findings across different types of stimuli and verbalizations. As stimuli we used male and female faces instead of letters to increase the complexity of stimuli as compared to the simpler letter version and to make the labeling of cues and probes less arbitrary and more familiar, and thus easier to apply for children and older adults. Male and female cues and probes were labeled as "Tom" or "Ruth," respectively.

\section{Method}

Participants. Overall, 25 children, 26 younger adults, and 27 older adults participated in the second experiment. We had to exclude three participants (one child and two older adults), as they had problems to follow the task instructions, and two participants (one younger and one older adult) because their error rates were larger than three standard deviations above their corresponding group mean. In addition, one younger adult was excluded due to task familiarity. This resulted in a final sample of 24 children (mean age $=9.0$ years, age range $=8-10$ years), 24 younger adults (mean age $=23.9$ years, age range $=20-33$ years), and 24 older adults (mean age $=71.8$ years, age range $=70-74$ years). Recruitment and reimbursement of the participants was the same as in Experiment 1. Also in the second experiment, except one younger adult, all of the participants were German native speaker. Participants reported themselves in good mental health (children: $M=1.6, S D=.72$; younger adults: $M=1.7, S D=.70$, older adults: $M=2.0, S D=.70$ ) as well as physical health (children: $M=1.6, S D=.50$; younger adults: $M=1.6, S D=.77$; older adults: $M=2.3, S D=.55)$. Again, younger and older adults did not differ in years of formal education $(p=.09)$.

As can be seen in Table 1 and similar to the findings in Experiment 1, we found age differences in speed of processing, $F(1,69)=152.0, p<.001, \eta_{\mathrm{p}}{ }^{2}=.815$, indicating that younger adults reached significantly higher scores than older adults, $F(1,69)=108.5, p<.001, \eta_{\mathrm{p}}^{2}=.611$, 
and older adults scored higher than children, $F(1,69)=47.6, p<.001, \eta_{\mathrm{p}}{ }^{2}=.408$. Moreover, young adults also reached significantly higher scores on the working-memory span task than older adults, $F(1,69)=12.1, p<.01, \eta_{\mathrm{p}}{ }^{2}=.149$, but older adults did not significantly differ from children, $F(1,69)=1.2, p=.277, \eta_{\mathrm{p}}^{2}=.017$. Finally, children and younger adults reached a lower score on the vocabulary subtest than older adults, $F(1,69)=302.5, p<.001, \eta_{\mathrm{p}}^{2}=.814$, and $F(1,69)=31.7, p<.001, \eta_{\mathrm{p}}^{2}=.315$, respectively.

Materials and procedure. Participants performed a modified version of the AX-CPT in which we used faces instead of letters. The visual stimuli consisted of eight standardized photographs of four different women and men, showing a neutral facial expression (adopted from the Karolinska Directed Emotional Faces database; Goeleven, De Raedt, Leyman, \& Verschuerg, 2008).

Participants had to press the p-key for AX-trials if the target female face occurred following the target male face, and the q-key for all other combinations of cue-probe presentations (nontarget cues or probes). The assignment of male and female faces to cue or probe was counterbalanced across subjects.

Similar to Experiment 1, participants were instructed to perform the labeling as a secondary task, that is, they were told to either name the cue or the probe stimulus. In the cuelabeling condition, they had to say aloud "Tom" if the cue was the male target face, whereas in reaction to all other cues they had to say "No." In the probe-labeling condition, they had to say aloud "Ruth" if the probe was the female target face, whereas in reaction to all other probes they had to say "No". In the control condition no labeling task had to be performed.

Procedure. The procedure was nearly identical to the first experiment. We only prolonged the presentation times for cues and probes to $800 \mathrm{~ms}$ and extended the response deadline to 1,500 ms. Moreover, all faces were presented to the participants before they 
performed the experimental task to familiarize them with the types of stimuli.

Data analysis. The rationale for data analysis was identical to the first experiment, except that RTs faster than $187 \mathrm{~ms}$ were excluded ( $0.3 \%$ of trials) from the analysis.

\section{Results}

Age differences in proactive and reactive control. The means and standard errors of the mean $(S E M s)$ of error rates and RTs are displayed in Figure 3 separately for each age group and the four trial types.

\section{- Insert Figure 3 about here-}

Errors. Results revealed that children made more errors than younger adults, $F(1,69)=$ $27.6, p<.001, \eta_{\mathrm{p}}{ }^{2}=.286$, but there was no reliable difference in error rates between younger and older adults, $F(1,69)=0.0, p=.872, \eta_{\mathrm{p}}^{2}=.000$. Like in Experiment 1, participants committed more errors on interference trials $(\mathrm{AY}, \mathrm{BX})$ than on noninterference trials $(\mathrm{AX}, \mathrm{BY}), F(1,71)=$ 47.7, $p<.001, \eta_{\mathrm{p}}{ }^{2}=.402$, and this effect was more pronounced for children than for younger adults, $F(1,69)=9.7, p<.01, \eta_{\mathrm{p}}{ }^{2}=.123$. As can be seen in Figure 3a, all participants made more errors on AY trials than on $\mathrm{BX}$ trials, $F(1,71)=31.0, p<.001, \eta_{\mathrm{p}}^{2}=.304$. Again in line with results from Experiment 1, age differences in the pattern of performance across AY and BX trials were absent.

$\boldsymbol{R T S .}$ In line with the results on error rates, children responded slower than younger adults, $F(1,69)=83.9, p<.001, \eta_{\mathrm{p}}^{2}=.549$, and older adults responded slower than younger adults, $F(1$, $69)=22.3, p<.001, \eta_{\mathrm{p}}{ }^{2}=.244$. Latencies were also slower on interference trials than on noninterference trials, $F(1,71)=107.6, p<.001, \eta_{\mathrm{p}}^{2}=.602$ and slower on AY than on BX trials, $F(1,71)=326.0, p<.001, \eta_{\mathrm{p}}^{2}=.821$, and this effect was larger for children than for younger adults, $F(1,69)=5.2, p<.05, \eta_{\mathrm{p}}^{2}=.070$. Again, the expected slowing of responses on $\mathrm{BX}$ trials for children and older adults, indicating reactive control, was not observed (see Figure 3b). 
Results did not change on the basis of log-transformed RT analysis.

The effects of cue and probe labeling. The means and SEMs for error rates and RTs are shown in Figure 4 as a function of labeling condition (control, cue labeling, probe labeling) and trial type (AY, BX) separately for each age group (children, younger adults, older adults).

Errors. As can be seen in Figure 4a, the pattern of findings is quite similar to the first experiment. Again, we found a larger increase in error rates under the cue labeling condition than under the control condition, $F(1,71)=8.7, p<.01, \eta_{\mathrm{p}}^{2}=.110$, and this increase was more pronounced for older than for younger adults, $F(1,69)=4.3, p<.05, \eta_{\mathrm{p}}^{2}=.059$. However, the increase in error rates was not significantly larger on AY trials than on $\mathrm{BX}$ trials, $F(1,71)=1.4, p$ $=.233, \eta_{\mathrm{p}}{ }^{2}=.020$, suggesting that the cue labeling did not specifically result in larger AY errors.

Error rates were also higher under probe labeling as compared to the control condition, $F(1,71)=10.0, p<.01, \eta_{\mathrm{p}}{ }^{2}=.123$, and again this effect was more pronounced for older than for younger adults, $F(1,69)=11.2, p<.01, \eta_{\mathrm{p}}^{2}=.139$. Like in Experiment 1, we found larger error rates on $\mathrm{BX}$ trials than on AY trials, $F(1,71)=20.5, p<.001, \eta_{\mathrm{p}}^{2}=.224$, but we did not obtain interactions with the two respective age contrasts, $F(1,69)=0.2, p=.704, \eta_{\mathrm{p}}^{2}=.002$, and $F(1$, 69) $=0.5, p=.506, \eta_{\mathrm{p}}^{2}=.006$.

Importantly, we found a significant interaction between labeling and trial type, $F(1,71)=$ $13.6, p<.001, \eta_{\mathrm{p}}^{2}=.161$, but no interaction with age, $F(2,69)=0.30, p=750, \eta_{\mathrm{p}}^{2}=.008$. Separate analysis for AY and BX trials showed an increase in BX errors under probe verbalization compared with cue verbalization, $F(1,71)=5.5, p<.05, \eta_{\mathrm{p}}^{2}=.071$, and a decrease of AY errors under probe verbalization, $F(1,71)=5.8, p<.05, \eta_{\mathrm{p}}^{2}=.076$ (see Figure $4 \mathrm{a}$ ).

RTs. Results showed slower latencies under cue labeling and probe labeling than under the control condition, $F(1,71)=12.7, p<.01, \eta_{\mathrm{p}}^{2}=.151$, and $F(1,71)=111.0, p<.001, \eta_{\mathrm{p}}^{2}=$ .610 , respectively. These effects were more pronounced for older adults than for younger adults, 
$F(1,69)=12.7, p=.053, \eta_{\mathrm{p}}{ }^{2}=.053$, and $F(1,69)=8.1, p<.01, \eta_{\mathrm{p}}{ }^{2}=.105$, respectively, but no interactions with trial type were found. However, as can be seen in Figure 4b, we obtained a significant interaction between labeling and trial type in line with the error data, $F(1,71)=23.4$, $p<.001, \eta_{\mathrm{p}}^{2}=.248$, indicating that latencies were slower under probe labeling than under cue labeling for BX trials than for AY trials (see Figure 4b), but this effect did not vary with age, $F(2$, 69) $=1.1, p=.328, \eta_{\mathrm{p}}^{2}=.031$.

\section{Discussion of Experiment 2}

Results of the second experiment are quite consistent with the findings obtained in the first experiment, although we increased the task complexity in the sense that subjects had to process more complex stimuli, namely faces instead of letters presented in the traditional AXCPT. Moreover, participants performed a more ecologically valid use of language by labeling faces with names instead of labeling letters. All age groups showed higher error rates and slower latencies on AY trials than on BX trials, suggesting that not only younger adults but also children and older adults were actively engaged in cue processing and advanced preparation. Furthermore, we did not find evidence that cue labeling further facilitated this processing mode in any age group. Like in Experiment 1, the results indicated a differential effect of probe labeling on the performance on BX trials. Thus, probe labeling indeed attracted the attention to the processing of the probe and lead to higher interference on trials in which the "Cue B" information had to be reactivated in order to overcome the bias of the frequent target response to the probe $\mathrm{X}$. At the same time AY errors decreased. The pattern of results clearly indicates a shift to a reactive mode for all age groups, in line with the first experiment. The results of Experiment 2 thus suggest that the reported findings are insensitive to changes in the kind of stimuli and types of verbal labeling, thereby contributing to the larger generalization of findings across context situations.

\section{Summary and General Discussion}


In this study we investigated in two experiments whether verbal labeling influences age differences in cognitive control processing and, in particular, whether it can promote flexible shifts between proactive and reactive control modes. We assessed three age groups (children, younger, and older adults) with a modified version of the AX-CPT task, which has been applied to examine individual differences in these control modes and instructed participants to different types of verbal labeling. We expected that cue labeling would enhance proactive control and probe labeling would enhance reactive control as labeling should attract the attention to different aspects of the underlying task.

\section{Age Differences in Proactive and Reactive Control}

Results of both experiments indicated that younger adults showed longer latencies and a higher error rate on AY trials than on BX trials and the two noninterference trials, indicating that they are highly engaged in updating and maintaining cue-relevant information, and by this are less sensitive to interference when the probe bias the incorrect response. This is in line with the view that younger adults primarily perform the task in a proactive control mode (Braver et al., 2001, 2009; Haarmann et al., 2005; Paxton et al., 2006, 2008; Rush et al., 2006). Children and older adults also showed longer latencies and a higher error rate on AY trials than on BX trials, quite similar to the pattern obtained in the younger age group. Hence, it seems that neither older adults nor children used a reactive control mode, as it has been suggested in previous developmental studies (Braver et al., 2009; Haarmann et al., 2005; Lorsbach \& Reimer, 2010; Paxton et al., 2006, 2008; Rush et al., 2006). However, it should be noted that the most convincing evidence for age differences in these control modes comes from neuroscientific developmental studies, reporting age-differential effects in the neuronal activation of specific brain regions, in particular, in the lateral PFC (Braver et al., 2009; Paxton et al., 2008), or agedifferential changes in high-resolution pupillometry measures during processing of the cue and 
probe information on AY and BX trials (cf. Chatham et al., 2009). Empirical findings on age differences in the pattern of performance is much more inconsistent at the behavioral level, where significant age differences are often restricted to either the error data or to the latency data (e.g., Lorsbach \& Reimer, 2010; Paxton et al., 2008; Rush et al. 2006). For instance, an increase of latencies on $\mathrm{BX}$ trials in younger children or an increase of error rates in older adults has sometimes been found only with very long cue-probe delays (above $5 \mathrm{~s}$ ) in which demands to maintain cue information are increased (cf. Lorsbach \& Reimer, 2010; Paxton et al., 2008, respectively). In contrast, much shorter cue-probe delays were applied in the present study.

While developmental studies on age differences in proactive and reactive control are scarce in childhood, more studies investigated age differences in these control modes at the other end of the lifespan. These studies found that, for instance, the amount of task practice or the reinstruction of the task can also induce a shift to a more proactive control mode in the elderly (Braver et al., 2009; Paxton, 2011; Paxton et al., 2006). We measured performance in the AXCPT under three different conditions and thus participants received more task practice than in other studies, and we also reinstructed the task rules for blocks requiring verbal labeling. These factors may have contributed to promoting a proactive control mode in children and older adults. Clearly more research is needed to disentangle the separate contributions of the different factors for the occurrence of age differences in proactive and reactive control.

\section{The Impact of Verbal Labeling on Age Differences in Cognitive Control}

The main question of the present study was whether different types of verbal labeling instructions can shift the attention toward cue or probe processing and by this influence age differences in the dynamic control of behavior. Given that all of our participants already performed the task in a proactive manner, cue labeling could not induce a shift from a reactive to a proactive control mode. Results of our study further showed that although cue labeling lead to 
an increase in errors and latencies on AY trials as compared conditions without verbal labeling, this increase was not larger as compared to BX trials or to probe labeling. Thus, cue labeling had no differential effect in further facilitating cue maintenance. Moreover, as the additional labeling lead to a slowing of responses, it might contradict the promotion of a proactive mode and has no specific effect on task performance. We know from recent studies, investigating the effects of task-goal labeling on the efficiency to switch between tasks, that benefits of verbal labeling (resulting in faster responding and smaller costs of switching) are restricted to situations in which the participants had already practice in task switching alone before performing the verbal labeling task in addition (Kray et al., 2010, 2013). Hence, we cannot exclude here that the selective effect of cue labeling would occur with more practice in the underlying task. On the other hand, probe labeling lead to a selective increase in errors on BX trials in both experiments. With probe labeling there was stronger interference on trials in which the relevant cue information had to be reactivated and participants had to overcome the response bias induced by the highly frequent target response to the probe $\mathrm{X}$. Hence, in line with our expectations, probe labeling resulted in a shift toward a more reactive control mode.

Moreover, this increase was even more pronounced for children than for younger and older adults, but only in the first experiment, in which children's mean age was lower than in the second experiment. Aside from this finding, the results of both experiments provided no evidence for age differences in the impact of verbal labeling on control functioning. So far these effects of verbal labeling have been mainly investigated with more demanding control tasks requiring the switching between often overlapping task rules, and different functions of language have been identified, such a serving as an important task retrieval aid (e.g., Kray et al., 2008). An important finding of this previous research is that especially children and older adults benefited from verbal labeling, suggesting that language can serve as a compensatory tool for the regulation of 
behavior. Altogether the findings seem to suggest that the compensatory function of language is restricted to the reconfiguration of cognitive processing between different tasks and does not extent to the configuration of processing within a given task. However, additional research is needed to confirm these considerations, for instance with prolonged cue-probe delays, best in combination with neuroscientific methods.

A further notable finding of the study is that the results highly generalized across different types of context conditions. Looking at the pattern of findings across the two experiments, the results were remarkably similar, although both experiments differed somewhat in the trial procedure, such as longer cue and probe presentation times and response deadlines in the second than in the first experiment, and they differed substantially in the stimulus materials and verbal labeling conditions. While the stimuli and verbal labeling instructions were more artificial in the first experiment, labeling someone's name when confronted with their face should have been more common to participants in the second experiment. Hence, the effects of verbal labeling on context processing seemed to be quite independent of task-specific contents.

In sum, the theoretical implications of the study are that the kind of verbal labeling can have a selective impact on the dynamic control of behavior: While cue labeling did not influence task-preparatory processing, that is, engagement in cue processing and maintenance, probe labeling influenced the ability to reactivate relevant cue information and to overcome a strong response bias during task execution. Our findings are also in line with theoretical ideas that the impact of labeling on task-preparatory processes is much smaller for transparent cues (cf. Chevalier \& Blaye, 2009) that directly trigger and bias response selection, as it is the case in the present study. So we do not know whether cue labeling may have induced a shift from a proactive to a reactive control mode for nontransparent or arbitrary cues.

Aside from theoretical implications what are practical implications of the present 
research? In general, the efficiency of cognitive control functioning and the dynamic control of behavior is known to be important for mastering a number of skills critical for academic and school success during childhood and also for intellectual functioning in older age. Therefore, cognitive interventions that can be easily instructed by specific kinds of task-supporting verbal labeling for a given task situation can be used as simple and powerful tools for promoting the control of behavior in various age groups without intensive training (cf. Kray \& Ferdinand, 2013). Such verbal labeling instructions can easily be applied by teachers, parents, and others in various task situations (e.g., homework, classroom) in order to redirect attention to task-relevant information. One challenge for future research, however, is not only to identify which kind of verbal labels are supportive for a given situation and why, but also to find out how to teach and remind individuals to apply them, if needed.

\section{Limitations of the Present Study}

Although results of our study were quite consistent across experiments, some limitations on its generalization should be noted. At first, to obtain a real lifespan perspective and to investigate shifts between proactive and reactive control in a more fine-grained manner across the lifespan, of course more age groups within childhood as well as adulthood should be included and longitudinal designs would be needed. In particular, the effects of labeling in promoting one mode of control or the other seems specifically worth testing in younger children based on recent findings with 5- to 6-year-old children, suggesting that 5 years of age could be a milestone in the developmental shift from reactive to proactive control (Lucenet \& Blaye, 2014). On the other hand, the occurrence of age differences in the AX-CPT seems to depend strongly on higher demands on cognitive control, such as working memory and interference demands (cf. Lorsbach \& Reimer, 2010). Moreover, it should be noted that our samples are often positively selected, including, for instance, university students or well-educated and physically healthy older adults 
Therefore, for a further generalization of findings, replications with other age ranges and samples are desirable.

A second limitation of the present study is that we did not include a task-irrelevant cue and probe verbalization condition or a motor task as additional control conditions in the present study. However, other studies have already demonstrated that verbal labeling effects are specific and produce different effects than task-irrelevant verbalization conditions (e.g., Kray et al., 2008). Moreover, as we predicted a differential effect of the labeling conditions on task performance and also used the corresponding other labeling condition as corresponding control condition, we can rule out that the effects are simply due to an increase in dual-task demands.

\section{Conclusion}

Results of this study suggest no age differences in dynamic control of tasks as measured with the AX-CPT. Instead, children, younger, and older adults were equally engaged in cue processing and actively prepared the task in advance and, thus, performed the task in a proactive manner. Verbal labeling, which has been considered a useful tool for the regulation of behavior, was used to selectively attract attention to task-relevant information. Cue labeling did not further promote a proactive control mode, probably because children and older adults, like younger adults, were already strongly engaged in cue processing. In the first study, children (who were younger in age than in the second study) were especially sensitive to the probe labeling instruction. Under probe labeling they were less able to maintain the cue information and to inhibit a strong response tendency induced by highly frequent events. Hence, results of the present study further support the view that verbal labeling has an impact on the regulation of behavior as it selectively affects the processing of the underlying task. Future studies need to clarify whether cue labeling can also promote a shift from a reactive to a proactive control mode by including even younger and older age samples or by increasing the demands on control 
processing.

\section{References}

Baltes, P. B., Staudinger, U. M., \& Lindenberger, U. (1999). Lifespan psychology: Theory and application to intellectual functioning. Annual Review of Psychology, 50, 471-507. doi:10.1146/annurev.psych.50.1.471

Braver, T. S. (2012). The variable nature of cognitive control: A dual mechanisms framework. Trends in Cognitive Sciences, 16, 106-113. doi:10.1016/j.tics.2011.12.010

Braver, T. S., \& Barch, D. M. (2002). A theory of cognitive control, aging cognition, and neuromodulation. Neuroscience and Biobehavioral Reviews, 26, 809-817. doi:10.1016/S0149-7634(02)00067-2

Braver, T. S., Barch, D. M., Keys, B. A., Carter, C. S., Cohen, J. D., Kaye, J. A., ... Reed, B. R. (2001). Context processing in older adults: Evidence for a theory relating cognitive control to neurobiology in healthy aging. Journal of Experimental Psychology: General, 130, 746763. doi:10.1037/0096-3445.130.4.746

Braver, T. S., Paxton, J. L., Locke, H. S., \& Barch, D.M. (2009). Flexible neural mechanisms of cognitive control within human prefrontal cortex. PNAS Proceedings of the National Academy of Sciences of the United States of America, 106, 7351-7356. doi:10.1073/pnas.0808187106

Bunge, S. A., \& Zelazo, P. D. (2006). A brain-based account of the development of rule use in childhood. Current Directions in Psychological Science, 15, 118-121. doi:10.1111/j.09637214.2006.00419.x

Chatham, C. H., Frank, M. J., \& Munakata, Y. (2009). Pupillometric and behavioral markers of a developmental shift in the temporal dynamics of cognitive control. PNAS Proceedings of the 
National Academy of Sciences of the United States of America, 106, 5529-5533. doi:10.1073_pnas.0810002106

Chevalier, N., \& Blaye, A. (2009). Setting goals to switch between tasks: Effect of cue transparency on children's cognitive flexibility. Developmental Psychology, 45, 782-797. doi:10.1037/a0015409

Cragg, L., \& Nation, K. (2010). Language and the development of cognitive control. Topics in Cognitive Science, 2, 631-642. doi:10.1111/j.1756-8765.2009.01080.

Dreisbach, G. (2006). How positive affect modulates cognitive control: The costs and benefits of reduced maintenance capability. Brain and Cognition, 60, 11-19. doi:10.1016/j.bandc.2005.08.003

Duncan, J. (1995). Attention, intelligence, and the frontal lobes. In M. S. Gazzaniga (Ed.), The cognitive neurosciences (pp. 721-733). Cambridge, MA: MIT Press.

Garon, N., Bryson, S. E., \& Smith, I. M. (2008). Executive function in preschoolers: A review using an integrative framework. Psychological Bulletin, 134, 31-60. doi:10.1037/00332909.134.1.31

Goeleven, E., De Raedt, R., Leyman, L., \& Verschuerg, B. (2008). The Karolinska Directed Emotional Faces: A validation study. Cognition and Emotion, 22, 1094-1118. doi:10.1080/02699930701626582

Haarmann, H. J., Ashling, G. E., Davelaar, E. J., \& Usher, M. (2005). Age-related declines in context maintenance and semantic short-term memory. The Quarterly Journal of Experimental Psychology A: Human Experimental Psychology, 58, 34-53. doi:10.1080/02724980443000214

Hofmann, W., Schmeichel, B. J., \& Baddeley, A. D. (2012). Executive functions and self-regulation. Trends in Cognitive Sciences, 16, 174-180. doi:10.1016/j.tics.2012.01.006 
Hughes, C. (2011). Changes and challenges in 20 years of research into the development of executive functions. Infant and Child Development, 20, 251-271. doi:10.1002/icd.736

Karbach, J., Mang, S., \& Kray, J. (2010). Transfer of task-switching training in older age: The role of verbal processes. Psychology and Aging, 25, 677-683. doi:10.1037/a0019845

Kray, J., Eber, J., \& Karbach, J. (2008). Verbal self-instructions in task switching: A compensatory tool for action-control deficits in childhood and old age? Developmental Science, 11, 223236. doi:10.1111/j.1467-7687.2008.00673.x

Kray, J. \& Ferdinand, N. K. (2013). How to improve cognitive control in childhood development: Potentials and limits of cognitive interventions. Child Development Perspectives, 7, 121125. doi:10.1111/cdep. 12027

Kray, J., Gaspard, H., Karbach, J., \& Blaye, A. (2013). Developmental changes in using verbal selfcueing in task-switching situations: The impact of task practice and task-sequencing demands. Frontiers in Psychology, 4: 940. doi:10.3389/fpsyg.2013.00940

Kray, J., Lucenet, J., \& Blaye, A. (2010). Can older adults enhance task-switching performance by verbal self-instructions? The influence of working-memory load and early learning. Frontiers in Aging Neuroscience, 2:147. doi:10.3389/fnagi.2010.00147

Lee, K., Ng, S. F., Pe, M. L., Ang, S. Y., Hasshim, M. N., \& Bull, R. (2012). The cognitive underpinnings of emerging mathematical skills: Executive functioning, patterns, numeracy, and arithmetic. British Journal of Educational Psychology, 82, 82-99. doi:10.1111/j.20448279.2010.02016.x

Lindenberger, U., Mayr, U., \& Kliegl, R. (1993). Speed and intelligence in old age. Psychology and Aging, 8, 207-220. doi:10.1037/0882-7974.8.2.207

Lorsbach, T. C., \& Reimer, J. F. (2008). Context processing and cognitive control in children and young adults. The Journal of Genetic Psychology: Research and Theory on Human 
Development, 169, 34-50. doi:10.3200/GNTP.169.1.34-50

Lorsbach, T. C. \& Reimer, J. F. (2010). Developmental differences in cognitive control: Goal representation and maintenance during a continuous performance task. Journal of Cognition and Development, 11, 185-216. doi:10.1080/15248371003699936

Lucenet, J., \& Blaye, A. (2014). Age-related changes in the temporal dynamics of executive control : A study in 5- and 6-year-old children. Manuscript submitted for publication.

Lucenet, J., Blaye, A., Chevalier, N., \& Kray, J. (2014). Cognitive control and language across the lifespan: Does labeling improve reactive control? Developmental Psychology, 50, 1620-1627. doi:10.1037/a0035867

Luria, A. R. (1961). The role of speech in the regulation of normal and abnormal behavior. Oxford, England: Liveright.

Miller, E. K., \& Cohen, J. D. (2001). An integrative theory of prefrontal cortex function. Annual Review of Neuroscience, 21, 167-202. doi:10.1146/annurev.neuro.24.1.167

Munakata, Y., Snyder, H. R., \& Chatham, C. H. (2012). Developing cognitive control: Three key transitions. Current Directions in Psychological Science, 21, 71-77. doi: $10.1177 / 0963721412436807$

Paxton, J. (2011). Effects of goal maintenance training on executive control in older adults. Electronic Theses and Dissertations, Paper 274. Retrieved from http://openscholarship.wustl.edu/etd/274

Paxton, J. L., Barch, D. M., Racine, C. A., \& Braver, T. S. (2008). Cognitive control, goal maintenance, and prefrontal function in healthy aging. Cerebral Cortex, 18, 1010-1028. doi:10.1093/cercor/bhm135

Paxton, J. L., Barch, D. M., Storandt, M., \& Braver, T. S. (2006). Effects of environmental support and strategy training on older adults' use of context. Psychology and Aging, 21, 499-509. 
doi:10.1037/0882-7974.21.3.499

Rush, B. K., Barch, D. M., \& Braver, T. S. (2006). Accounting for cognitive aging: Context processing, inhibition, or processing speed? Neuropsychology, Development, and Cognition, 13, 588-610. doi:10.1080/13825580600680703

Smith, E. E., \& Jonides, J. (1999). Storage and executive processes in the frontal lobes. Science, 283, 1657-1661. doi:10.1126/science.283.5408.1657

Vygotsky, L. S. (1962). Thought and language. Cambridge, MA: MIT Press. .doi:10.1037/11193000

Wechsler D. (2003). Wechsler Intelligence Scale for Children (4 $4^{\text {th }}$ ed.). San Antonio, TX: Psychological Corporation.

West, R., \& Schwarb, H. (2006). The influence of aging and frontal function on the neural correlates of regulative and evaluative aspects of cognitive control. Neuropsychology, 20, 468-481. doi:10.1037/0894-4105.20.4.468

Zelazo, P. D., \& Frye, D. (1997). Cognitive complexity and control: A theory of the development of deliberate reasoning and internal action. In M. Stamenov (Ed.), Language structure, discourse, and access to consciousness (pp. 113-153). Amsterdam, The Netherlands: John Benjamin. 


\section{Footnotes}

${ }^{1}$ We used the paper-and-pencil version of the Digit Symbol Substitution Test to assess processing speed. Subjects were presented a sheet which contained three lines of numbers from 1 to 9 in a random order. A template on the top showed which number belonged to one out of nine different symbols. The task was to draw as quickly and as accurately as possible the correct symbol below each number. The score was the number of correctly combined symbols within 90 s (maximum value 93).

The backward digit-span task assesses working memory. Participants were orally presented a sequence of digits ranging from 4 to 8 and had to repeat aloud each sequence in the reversed order of presentation. Three sequences for each span were given and the score of the participants was the number of items that were correctly repeated in the reversed order (maximum value 15).

The Spot-a-Word test measured vocabulary, that is, crystallized intelligence. The test consisted of 35 items, which were presented successively on a computer screen. Each item consisted of one word and four nonwords, and subjects had to select the genuine word. The test score was the total number of correct words found.

${ }^{2}$ We also analyzed incorrect responses alone, and it confirmed our results of incorrect responses including omissions. 
Table 1

Sample Characteristics and Means (Standard Errors) of Cognitive Variables for Children, Younger, and Older Adults in Experiments 1 and 2

\begin{tabular}{|c|c|c|c|}
\hline Variables & Children & Younger adults & Older adults \\
\hline & \multicolumn{3}{|c|}{ Experiment 1} \\
\hline$N$ & 18 & 18 & 16 \\
\hline$\%$ female & 50 & 50 & 78 \\
\hline Age range & $7-8$ & $19-30$ & $69-83$ \\
\hline Mean age & $7.2(0.4)$ & $22.5(3.4)$ & $74.4(4.1)$ \\
\hline Digit-symbol test & $28.4(5.4)$ & $69.4(9.7)$ & $48.1(10.3)$ \\
\hline Digit-span test & $3.7(1.7)$ & $9.4(1.9)$ & $6.6(2.1)$ \\
\hline \multirow[t]{2}{*}{ Spot-a-word test } & $9.0(2.4)$ & $22.9(1.7)$ & $27.8(2.3)$ \\
\hline & \multicolumn{3}{|c|}{ Experiment 2} \\
\hline$N$ & 24 & 24 & 24 \\
\hline$\%$ female & 50 & 50 & 50 \\
\hline Age range & $8-10$ & $20-33$ & $70-74$ \\
\hline Mean age & $9.0(0.6)$ & $23.9(3.4)$ & $71.8(1.2)$ \\
\hline Digit-symbol test & $30.5(4.6)$ & $68.6(9.1)$ & $45.7(8.5)$ \\
\hline Digit-span test & $4.9(2.1)$ & $7.8(2.1)$ & $5.6(2.4)$ \\
\hline Spot-a-word test & $11.7(2.3)$ & $22.7(4.7)$ & $28.0(2.1)$ \\
\hline
\end{tabular}




\section{Figure captions}

Figure 1. (a) Means and SEMs of the error rates (in \%) as a function of trial type (AX, AY, BX, BY) and age group (children, younger adults, older adults), and (b) means and SEMs of the RTs (in ms) as a function of trial type (AX, AY, BX, BY) and age group (children, younger adults, older adults) in Experiment 1.

Figure 2. (a) Means and SEMs for error rates (in \%) as a function of trial type (AY, BX) and verbalization condition (control, cue verbalization, probe verbalization) separately for children (left panel), younger adults (middle panel), and older adults (right panel); and (b) means and SEMs for RTs (in ms) as a function of trial type (AY, BX) and verbalization condition (control, cue verbalization, probe verbalization) separately for children (left panel), younger adults (middle panel), and older adults (right panel) in Experiment 1.

Figure 3. (a) Means and SEMs of the error rates (in \%) as a function of trial type (AX, AY, BX, BY) and age group (children, younger adults, older adults), and (b) means and SEMs of the RTs (in ms) as a function of trial type (AX, AY, BX, BY) and age group (children, younger adults, older adults) in Experiment 2.

Figure 4. (a) Means and SEMs for error rates (in \%) as a function of trial type (AY, BX) and verbalization condition (control, cue verbalization, probe verbalization) separately for children (left panel), younger adults (middle panel), and older adults (right panel); and (b) means and SEMs for RTs (in ms) as a function of trial type (AY, BX) and verbalization condition (control, cue verbalization, probe verbalization) separately for children (left panel), younger adults (middle panel), and older adults (right panel) in Experiment 2. 
(a)
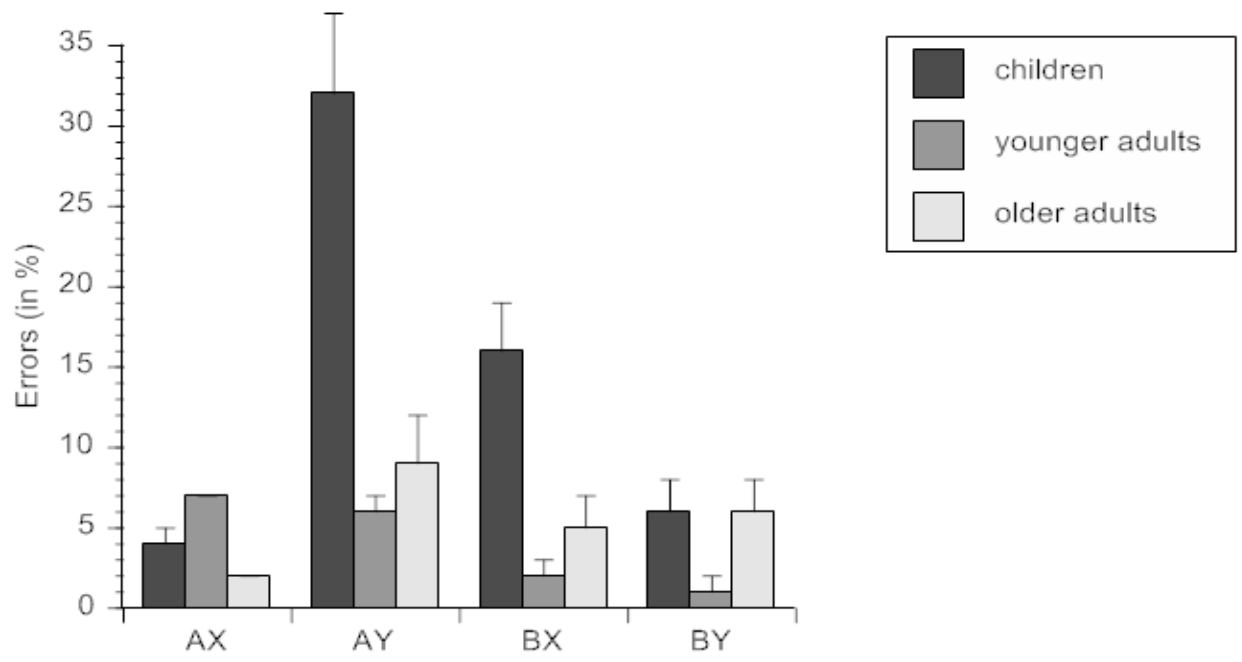

(b)
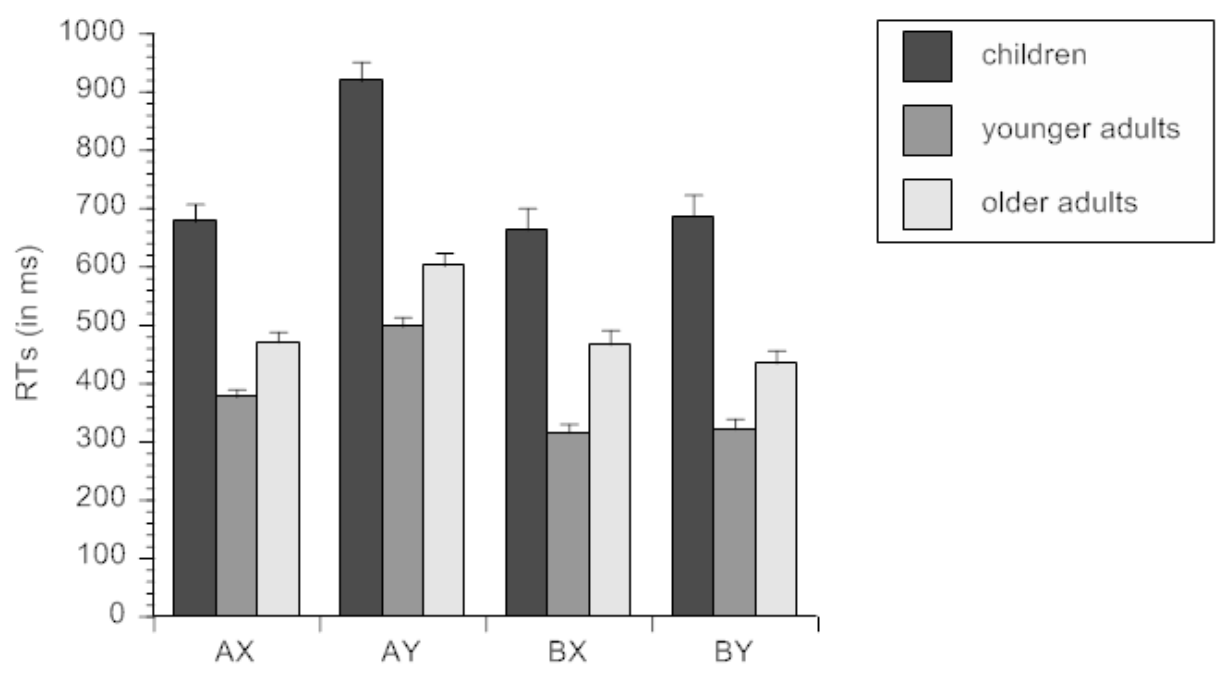

Figure 1 

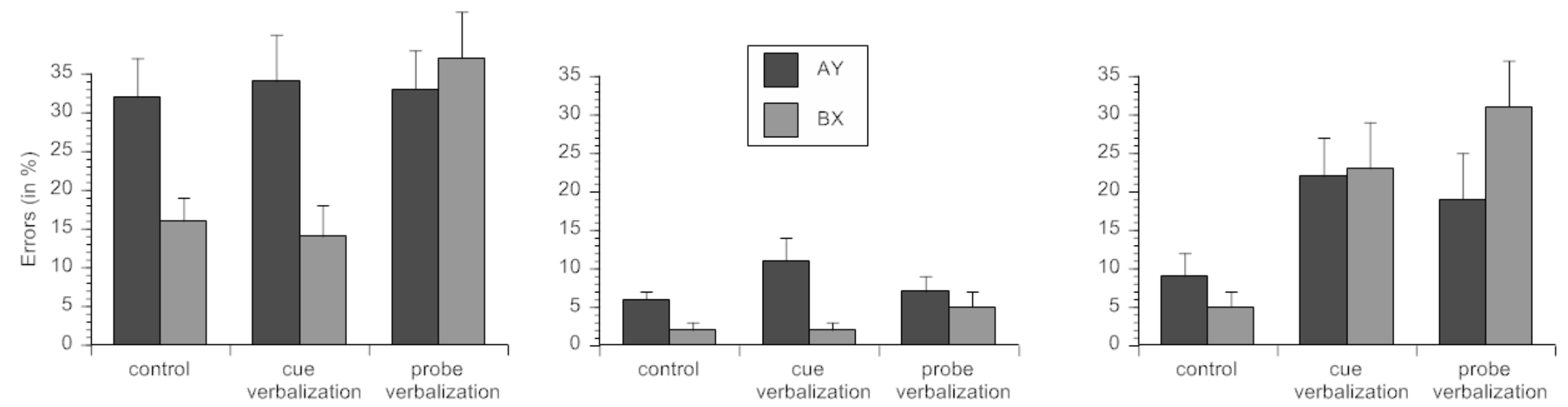

\section{b) Reaction times}
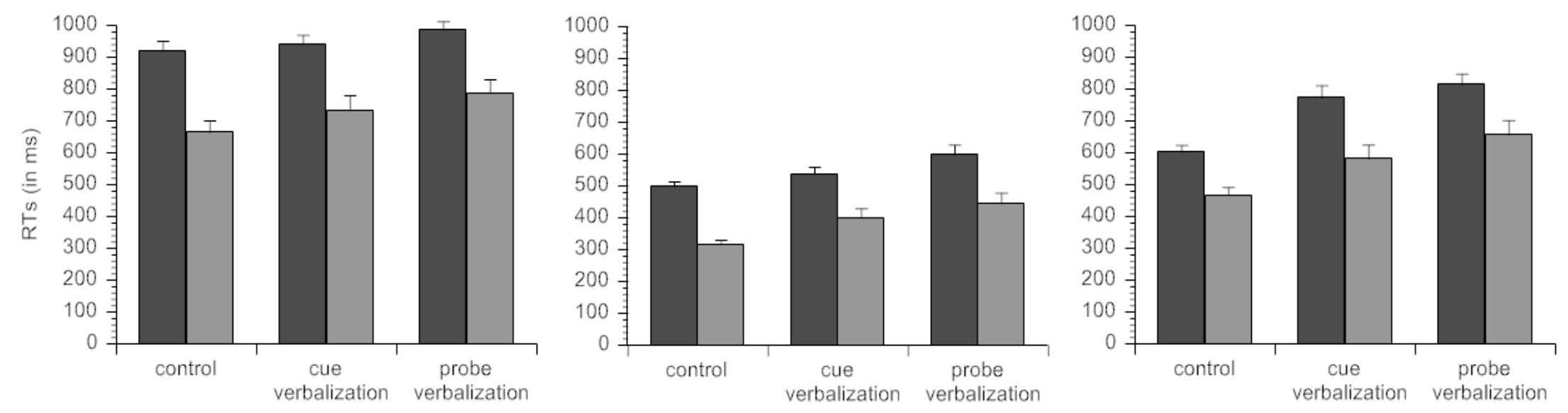

Figure 2 
(a)
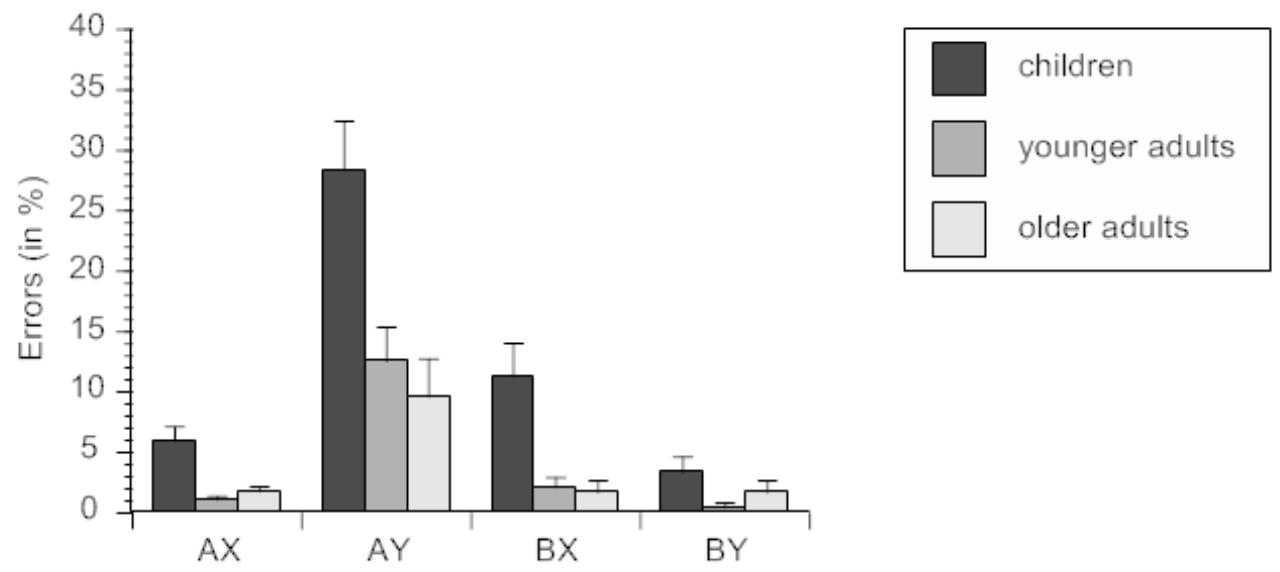

(b)

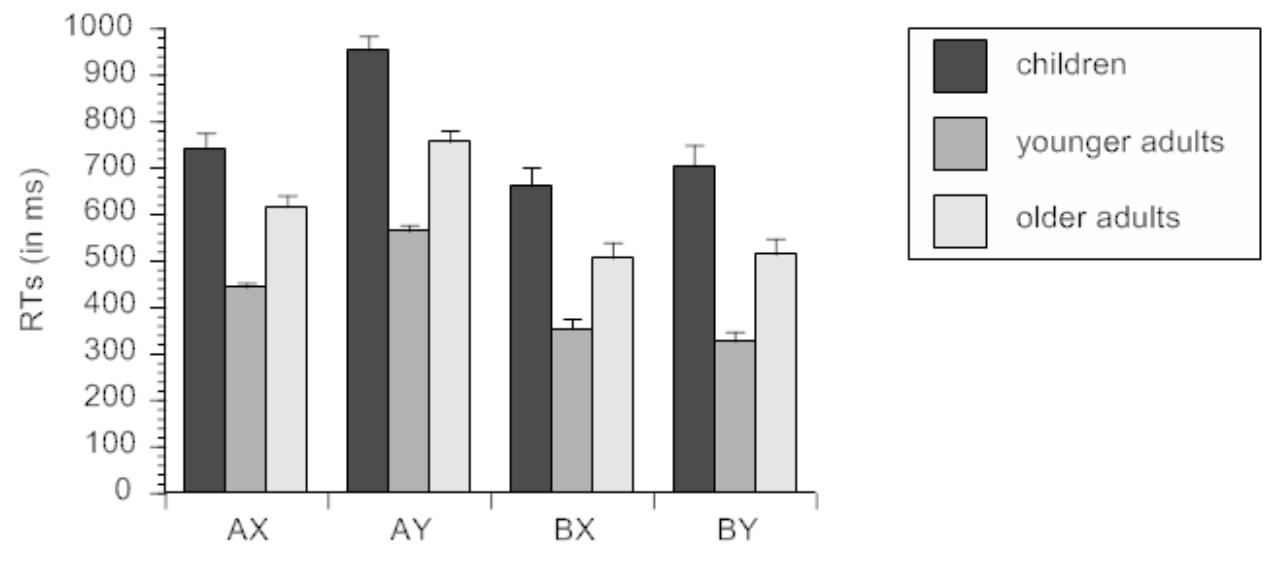

Figure 3 
(a)
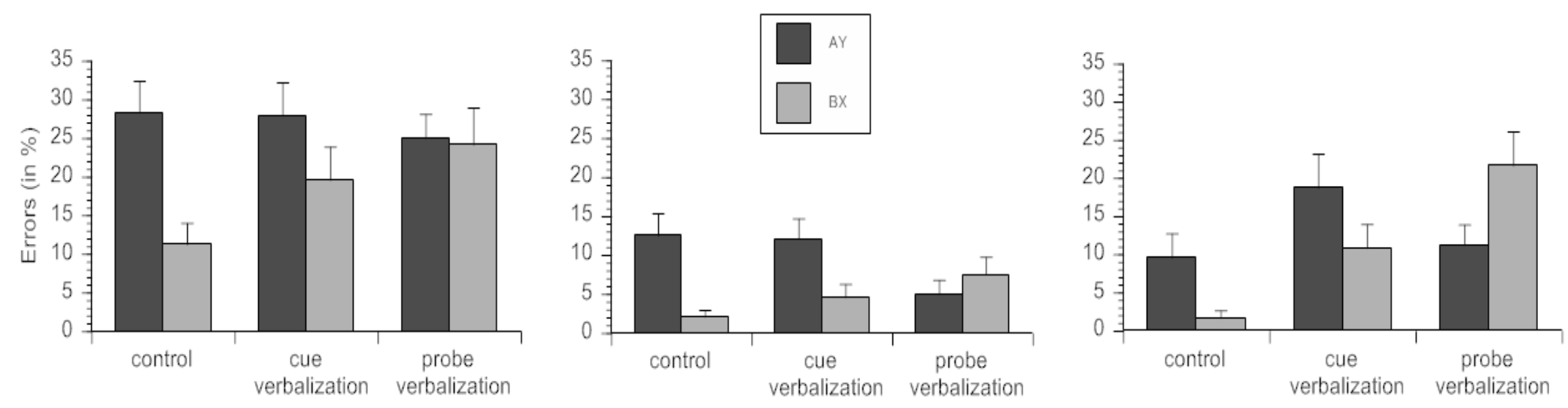

(b)
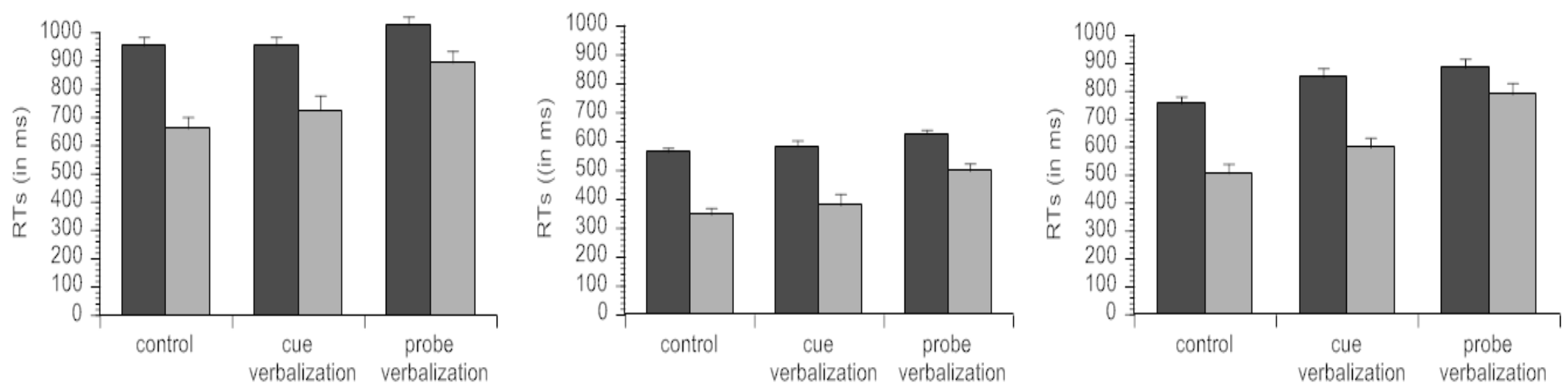

Figure 4 